\title{
Generation of high-frequency bunched electrons from photoinjector with a multiring photocathode
}

\author{
Fu-Han Chao $\odot,{ }^{1}$ Chia-Hsiang Chen, ${ }^{2,3}$ Ping-Jung Chou $\odot,{ }^{1,2}$ and Yen-Chieh Huang $\odot^{1,3, *}$ \\ ${ }^{1}$ Department of Physics, National Tsing Hua University, Hsinchu 300044, Taiwan \\ ${ }^{2}$ National Synchrotron Radiation Research Center, Hsinchu 30076, Taiwan \\ ${ }^{3}$ HOPE Laboratory, Institute of Photonics Technologies, National Tsing Hua University, \\ Hsinchu 300044, Taiwan
}

(Received 4 October 2020; revised 3 April 2021; accepted 27 April 2021; published 27 May 2021)

\begin{abstract}
In this paper, we propose to manipulate a nonrelativistic electron beam during its emission and acceleration to create nanobunched electrons. Specifically, we simulate the acceleration of a structured electron beam emitted from a multiring photocathode in a photoinjector and show that, after acceleration, such a transversely structured beam can be transformed into a hollow beam with a longitudinal density modulation in the petahertz frequency range. Our theoretical study shows that, in the far-field zone, the coherent radiation of the bunched hollow beam approaches that of a similarly bunched solid beam in the paraxial limit.
\end{abstract}

DOI: 10.1103/PhysRevAccelBeams.24.052801

\section{INTRODUCTION}

It is well known that an electron beam having a length much longer than the desired radiation wavelength emits incoherent radiation with spectral energy linearly proportional to the number of electrons in the beam. However, when the electron bunch is much shorter than the radiation wavelength, the bunch can emit coherent radiation with spectral energy proportional to the square of the number of electrons in the bunch [1-3]. This coherent radiation process is dubbed as superradiant emission or superradiance. For an electron bunch with a finite length, the radiation intensity is scaled by the Fourier amplitude of the bunch or the so-called bunching factor. Furthermore, a density-modulated electron beam consisting of $N_{b}$ periodic bunches could radiate coherently at the harmonics of its bunching frequency with spectral energy proportional to $N_{b}{ }^{2}$. It is possible to inject a coherent seed radiation into a superradiant process to generate more radiation power through stimulated emission. This is called the stimulated superradiant process [1-3]. Superradiant and stimulatedsuperradiant emissions of longitudinally density-modulated electron beams are useful to generate intense coherent radiations from, for instance, Smith-Purcell grating [4-7], Cherenkov dielectric [8,9], transition radiator [10-12], and free electron laser (FEL) [13-27].

\footnotetext{
ychuang@ee.nthu.edu.tw
}

Published by the American Physical Society under the terms of the Creative Commons Attribution 3.0 License. Further distribution of this work must maintain attribution to the author(s) and the published article's title, journal citation, and DOI.
To achieve multibunch superradiance, the preparation of a density-modulated beam is imperative. To efficiently generate superradiant or stimulated superradiant emission, the length of each microbunch in the beam has to be comparable to or shorter than the desired radiation wavelength. For example, a Gaussian bunch with its rms length equal to half of the radiation wavelength gives a bunching factor of about $1 \%$ [28]. For a periodically bunched beam, the repetition rate of the microbunches should be the same as or a subharmonic of the desired radiation frequency. It is increasingly difficult to generate high-frequency bunching toward terahertz, not to mention the extreme ultraviolet (EUV) or soft x-ray frequencies. In the EUV and soft x-ray spectrum, the bunching frequency has to be in the range of few tens or even few hundreds of petahertz $(\mathrm{PHz})$, which has a corresponding bunch duration in the range of few tens or even few attoseconds. Although a high-voltage dc electron gun followed by superconducting radio frequency (SRF) cavities is able to generate a bunch repetition rate up to a few $\mathrm{GHz}$ [29-31], it is far below what is needed for generating $\mathrm{THz}, \mathrm{EUV}$ or soft $\mathrm{x}$-ray superradiance.

Many schemes of laser-based manipulation have been proposed for creating microstructures in an electron beam. The laser-pulse train driven photoemission was proposed to generate electron bunches with a repetition rate up to few hundreds of $\mathrm{THz}[28,32]$. It has been experimentally demonstrated that two electron bunches separated by few ps with total charges of a few tens of $\mathrm{pC}$ can be generated from a radio frequency (rf) photoinjector driven by sub-ps laser pulses [32]. Besides, the interaction between a relativistic electron beam and the field of a laser in a modulator undulator can imprint an energy modulation on 
an electron beam, which can then be converted into a longitudinal density modulation in the beam. Some of these phase-space manipulation schemes, such as high-gain harmonic generation (HGHG) [16-19] and echo-enabled harmonic generation (EEHG) [18-27], have been used to bunch a beam to enhance the coherent radiation in an FEL. Recently, coherent radiations at 4.3 [16] and $32 \mathrm{~nm}$ [27] have been achieved with HGHG and EEHG schemes, respectively. Nevertheless, the interaction between the electron beam and the field of a laser often involves complex dispersive elements, such as undulators or cascaded modulator-chicane modules. It has also been proposed to insert a mask into a beam line to create transverse structures in a beam, and then convert them into longitudinal structures through the so-called transverse-tolongitudinal emittance exchange (EEX) scheme [33-37]. The longitudinal period between the microbunches generated by the EEX scheme is usually between sub-ps and ps [33-37], which corresponds to a bunching frequency in the $\mathrm{THz}$ region. The EEX scheme also involves dispersive elements, which usually induce unwanted beam degradation from incoherent and coherent synchrotron radiations [23]. In this paper, we propose a novel bunching scheme that does not involve dispersive elements and seed lasers.

Similar to the phase space manipulation of a relativistic electron beam in dispersive elements, a nonrelativistic electron beam can be manipulated with ease by the rf field in an accelerator cavity. In Sec. II, we introduce the spatial transformation of a short electron bunch by the radially dependent rf fields in an accelerator cell. We will discuss the influence of the injection phase on the bunch compression in an $\mathrm{rf}$ photoinjector. By tailoring the transverse bunch distribution at the photocathode, one can utilize the spatial transformation of the electron beam in rf cavities to precisely modulate the electron density in the longitudinal direction during particle acceleration. An important advantage of the proposed scheme is that there is no dispersive element along the beam line to cause beam degradation. Previously, a curved cathode was proposed to cancel the radial-path difference among emitted electrons and generate a single ultrashort electron bunch [38]. In this work, we take advantage of the path difference on a structured flat cathode to generate a fast electron bunch train after acceleration. In Sec. III, we introduce a key parameter, the bunching factor, for qualifying the bunching of the beam. We then introduce the particular form it takes under the paraxial approximation in the far-field region, as well as its simplification to a one-dimensional (1D) longitudinal bunching factor for certain beam properties. In Sec. IV, we propose to use the aforementioned spatial transformation of electrons to generate a longitudinally density modulated electron beam by illuminating a multiring cathode of a photoinjector with a laser pulse. We simulate the particle dynamics in a normal conducting rf (NCRF) accelerator system by using the particle tracking code ASTRA [39].
The simulation results show that the transversely structured beam can be spatially transformed into a hollow beam with longitudinal density modulation during particle acceleration. Under certain conditions, the bunching frequency can reach the $\mathrm{PHz}$ range, although efficient bunching in the $\mathrm{THz}$ range could already been useful. We also analytically calculate and compare the bunching factors of a hollow and a solid beam with the same longitudinal density modulation. As it will be shown below, the comparison indicates that the far-field coherent radiation of the hollow beam generated by our scheme is similar to that of a solid beam in the paraxial limit.

\section{RADIAL DEPENDENCE OF rf ACCELERATION}

\section{A. Radially dependent $\mathbf{r f}$ accelerating fields}

The radially dependent electric fields in an accelerator account for the coupling of the radial and longitudinal displacements of the electrons in the beam during acceleration. In this paper, the longitudinal direction is defined along $z$, coinciding with the electron propagation direction. In a cylindrical pillbox rf cavity, the longitudinal accelerating field has its maximum value on the axis of the accelerator. In the radial direction, the longitudinal field decreases gradually and vanishes at the transverse sidewall of the cavity. On the other hand, the radial field strength increases along the radial direction. Specifically, for an accelerating cavity with a length $L$ and radius $a$, the longitudinal and radial electric-field amplitudes of the fundamental cavity mode are given by [14]

$$
E_{z 0}(r)=E_{0} J_{0}\left(\eta_{0} r\right)
$$

and

$$
E_{r 0}(r)=\frac{k_{0}}{\eta_{0}} E_{0} J_{1}\left(\eta_{0} r\right),
$$

respectively, where $J_{0}$ and $J_{1}$ are the zeroth and first order Bessel functions of the first kind, $E_{0}$ is the maximum axial field amplitude, $\eta_{0}=2.405 / a$ and $k_{0}=\pi / L$. Both of the fields in Eqs. (1) and (2) are dependent on $r$. For an S-band $\pi / 2$-mode standing-wave accelerator operating at $2.856 \mathrm{GHz}$ with $L=6.76 \mathrm{~cm}$ and $a=4.2 \mathrm{~cm}, k_{0}=$ 46.47 and $\eta_{0}=57.16$. Figure 1 shows the variations of $E_{z 0}(r) / E_{0}$ and $E_{r 0}(r) / E_{0}$ along $r$ in the paraxial region of such an rf cavity. Note that the longitudinal acceleration gradient $E_{z 0}$ decreases to about $99.67 \%$ of the maximum value $E_{0}$ at the radial position $r=2 \mathrm{~mm}$. As will be seen below, this small change of the accelerating field is sufficient to introduce a significant spatial transformation to a beam at the exit of an accelerator.

In addition to the accelerating field, the space charge field of the beam also influences the motion of the electrons in the beam. With a few-cycle fs injection laser, it is 


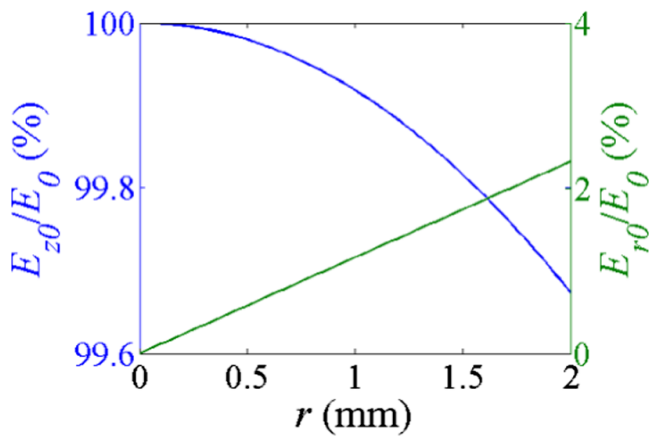

FIG. 1. Variations of $E_{z 0} / E_{0}$ and $E_{r 0} / E_{0}$ versus $r$ for $k_{0}=$ 46.47 and $\eta_{0}=57.16$ in an S-band accelerator cell.

possible to induce the emission of an ultrashort electron bunch at the photocathode. Considering a longitudinally thin electron disk with a surface charge density $\rho_{s}$, near the center of the disk, the space charge field is roughly $\rho_{s} / 2 \varepsilon_{0}$ at both the front and back sides of the electron layer, where $\varepsilon_{0}$ is the vacuum permittivity. For a 100 -fC electron beam with an initial Lorentz factor $\gamma \sim 1$ uniformly distributed over a two-dimensional disk within a radial distance $r=2 \mathrm{~mm}$, the space charge field at the center of the disk surface is about $E_{\mathrm{sc}} \sim 449.39 \mathrm{~V} / \mathrm{m}$, which is significantly smaller than the nominal tens of $\mathrm{MV} / \mathrm{m}$ accelerating field in an accelerator. However, if the total charge increases to $1 \mathrm{nC}$ over the same disk area, the space charge field is comparable to the accelerating field.

The dynamics of a single electron in a standing-wave $\mathrm{rf}$ accelerator has been studied in [40] without considering the space charge and transverse rf fields. From the approximate solutions, the rf phase seen by an electron, $\phi \equiv$ $\omega t-k_{0} z+\phi_{0}$, has the asymptotic value, $\phi \rightarrow \phi_{\infty}=$ $\left(m_{0} c^{2} k_{0} / e E_{z 0} \sin \phi_{0}\right)+\phi_{0}$, subject to an accelerating field $E_{z 0}$, where $\phi_{0}$ is the initial phase of the accelerating field, $m_{0}$ is the electron rest mass, and $e$ is the electron charge. The asymptotic phase difference between two electrons generated at $r=r_{1}$ and $r_{2}$ is $\phi_{\infty}\left(r=r_{1}\right)-\phi_{\infty}\left(r=r_{2}\right)=$ $m_{0} c^{2} k_{0} / e E_{z 0}\left[J_{0}\left(\eta_{0} r_{1}\right)-J_{0}\left(\eta_{0} r_{2}\right)\right] \sin \phi_{0}$. The phase difference indicates that the radially dependent field $E_{z 0}(r)$ can longitudinally separate the two electrons during acceleration. However, this approximate solution is not valid for $\sin \phi_{0} \rightarrow 0$. In addition, the model is too complicated to give a close-form solution when involving the transverse $\mathrm{rf}$ fields [28]. To take into account the effects of the space charge and transverse rf fields for high-frequency bunching, for what follows, we carefully study the details in computer simulations.

\section{B. Spatial transformation of a short electron beam}

A short electron beam emitted from a photocathode can be regarded as a longitudinally thin particle layer with a finite radial extent, if its transverse dimension is much larger than the longitudinal dimension. Since the rf acceleration force experienced by an electron is radially dependent in an rf accelerator, as mentioned in Sec. II A, the electron layer is spatially transformed into a different geometry during acceleration [28]. We propose to use this effect to create a longitudinal density modulation with an initially transversely structured beam. As it will be shown in Sec. IV, we first investigate here the possibility to keep the particle layer thin during the acceleration by a proper choice of the injection phase in an rf photoinjector.

At the photocathode, the initial thickness of the particle layer is mostly determined by the pulse duration of the driver laser. When an electron leaves from the cathode surface, it experiences simultaneously an rf acceleration force and a repulsive space charge force from the other electrons. Hence, the thickness of a subsequently accelerated particle layer depends not only on the laser pulse duration and the space charge force, but also on the injection phase and acceleration-field gradient of the $\mathrm{rf}$ photoinjector. The beam compression factor, which is defined in the paper as the ratio of the final temporal spread to the initial temporal width of particles at the same

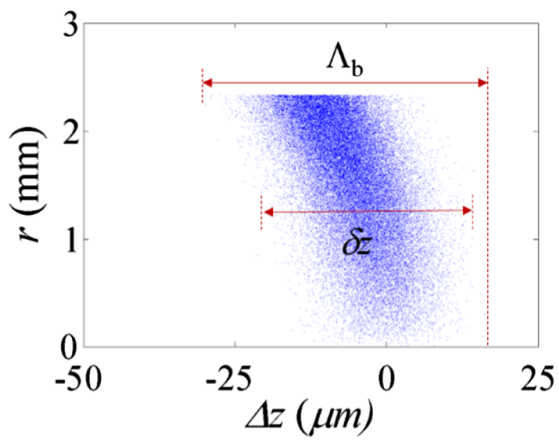

(b)

FIG. 2. Half $r-\Delta z$ distribution of 100-fC electron beams at the exit of the 1.6-cell, S-band rf photoinjector [42] with $E_{0}=100 \mathrm{MV} / \mathrm{m}$. The injection phases are (a) $180^{\circ}$ and (b) $200^{\circ}$, respectively. Note that the initial cutoff radius and temporal duration of the beams are $2 \mathrm{~mm}$ and $30 \mathrm{fs}$ at the photocathode, respectively. $\delta z, \Lambda_{b}, \Delta z$ are the spread of the particle layer, full length of the electron beam, and longitudinal particle position with reference to a particle on the axis, respectively. 
radial position in an rf photoinjector, was analytically studied in [41]. The analysis indicates that the beam compression factor for negatively charged particles attains a minimal value when the injection phase of the rf photoinjector is $180^{\circ}$. As a result, the rf fields could compress the electron bunch during acceleration when the injection phase is around $180^{\circ}$. Note that at $180^{\circ}$ the phase of the longitudinal rf field on the cathode changes its sign. For negatively charged particles, the rf field is accelerating at $180^{\circ}$ and deaccelerating at $0^{\circ}$. Figures 2 (a) and 2(b) show typical ASTRA simulation results of short electron beams generated from a $100 \mathrm{MV} / \mathrm{m}$ BNL/SLAC/UCLA-type 1.6-cell, S-band rf photoinjector [42] with injection phases at $180^{\circ}$ and $200^{\circ}$, respectively. For the results shown in the plot, the electron beams are set to be uniformly distributed in the transverse direction with a cutoff radius $r_{0}=2 \mathrm{~mm}$ at the cathode. The total charge of the electron beams and the rms pulse duration of the driver laser are $100 \mathrm{fC}$ and $30 \mathrm{fs}$, respectively. The electron beam in Fig. 2(a), although lengthened to $\Lambda_{b}$ (the full longitudinal length) under the influence of the radially dependent $\mathrm{rf}$ accelerating fields, remains a thin layer with a longitudinal sliced thickness denoted by $\delta z$. It is seen that the thickness of a nonrelativistic electron layer is sensitive to the injection phase of the rf photoinjector. In fact, at the $200^{\circ}$ injection phase, the layer thickness $\delta z$ increases significantly from its initial value, which is undesirable for electron bunching.

\section{RADIATION AND BUNCHING FACTOR OF DENSITY MODULATED BEAM}

The bunching factor $M_{b}$ crucially weights the coherent radiation power emitted by a beam. The angular spectral energy emitted by a beam containing $N$ electrons in a radiator can be expressed as [43]

$$
\begin{aligned}
\frac{d^{2} W}{d \Omega d f} & =\left(\frac{d^{2} W}{d \Omega d f}\right)_{\mathrm{inc}}+\left(\frac{d^{2} W}{d \Omega d f}\right)_{\mathrm{coh}} \\
& \cong N\left[1+N M_{b}^{2}(\Omega, f)\right]\left(\frac{d^{2} W}{d \Omega d f}\right)_{0},
\end{aligned}
$$

where $\Omega$ is the solid angle with reference to the $z$ axis, $M_{b}(\Omega, f)$ is the bunching factor as a function of the solid angle $\Omega$ and frequency $f,\left(d^{2} W / d \Omega d f\right)_{0}$ is the angular spectral energy radiated by a single electron, $\left(d^{2} W / d \Omega d f\right)_{\text {inc }}=N\left(d^{2} W / d \Omega d f\right)_{0}$ and $\left(d^{2} W / d \Omega d f\right)_{\text {coh }}=$ $N^{2} M_{b}^{2}(\Omega, f)\left(d W^{2} / d \Omega d f\right)_{0}$ are the incoherent and coherent parts of the energy emitted by the beam, respectively. Given the enormous number of electrons in a beam, even a small bunching factor would allow the coherent radiation energy from a bunched beam exceeding the incoherent one from a randomly distributed beam. For example, it is straightforward to show from Eq. (3) that the radiation energy from a 100-fC density modulated beam with $M_{b}=$ 0.04 can be as high as the one from a 100-pC randomly distributed beam.

\section{A. Bunching factor of an arbitrarily shaped electron beam}

Consider an observer at the position $\vec{R}=(x, y, d)$ and an electron beam with an arbitrary shape longitudinally extended between $z=-\Lambda_{b}$ and $z=0$, as illustrated in Fig. 3. We define the observation angle as $\theta \cong \sqrt{x^{2}+y^{2}} / d$ with $\sqrt{x^{2}+y^{2}} \ll d$ in the paraxial limit $\theta \ll 1$. Assuming that the distance $d$ between the observer and the beam head is much larger than the beam length $\Lambda_{b}$, and taking the beam's three-dimensional (3D) distribution into account, one can express the bunching factor as [43]

$$
\begin{aligned}
M_{b}(x, y, f) & \equiv \frac{1}{N}\left|\sum_{j=1}^{N} \exp \left(i \frac{2 \pi f\left|\vec{R}-\overrightarrow{R_{j}}\right|}{c}\right)\right| \\
& \cong \frac{1}{N}\left|\sum_{j=1}^{N} \exp \left\{i \frac{2 \pi f\left(d-z_{j}^{\prime}\right)}{c}+i \frac{\pi f\left[\left(x-x_{j^{\prime}}\right)^{2}+\left(y-y_{j^{\prime}}\right)^{2}\right]}{c\left(d-z_{j}^{\prime}\right)}\right\}\right|
\end{aligned}
$$

where $\overrightarrow{R_{j}^{\prime}}=\left(x_{j}^{\prime}, y_{j}^{\prime}, z_{j}^{\prime}\right)$ represents the position of the $j$ th electron. The approximation in Eq. (4) is valid only if the paraxial approximation $\left(x-x_{j}^{\prime}\right)^{2}+\left(y-y_{j}^{\prime}\right)^{2} \ll\left(d-z_{j}^{\prime}\right)^{2}$ is satisfied. The inverse of the longitudinal distance between the observer and the $j$ th electron $\left(d-z_{j}^{\prime}\right)^{-1}=1 / d\left[1+z_{j}^{\prime} / d+\left(z_{j}^{\prime} / d\right)^{2}+\left(z_{j}^{\prime} / d\right)^{3}+\cdots\right]$ can be approximated as $1 / d$ within the range $-\Lambda_{b} \leq z_{j}^{\prime} \leq 0$, since $\Lambda_{b} / d \ll 1$. The bunching factor in Eq. (4) depends not only on the particle distribution in the beam but also on the position of the observer relative to the beam. By taking out the constant phase terms, Eq. (4) can be rewritten as

$$
M_{b}(x, y, f) \cong \frac{1}{N}\left|\sum_{j=1}^{N} \exp \left[-i \frac{2 \pi f z_{j}^{\prime}}{c}-i \frac{2 \pi f\left(x x_{j}^{\prime}+y y_{j}^{\prime}\right)}{c d}+i \frac{\pi f\left(x_{j}^{\prime 2}+y_{j}^{\prime 2}\right)}{c d}\right]\right|
$$




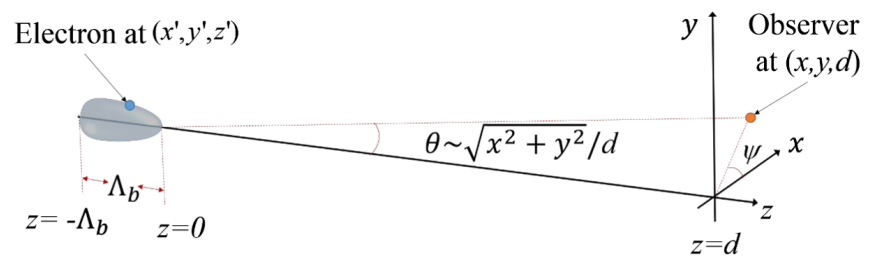

FIG. 3. Coordinates for calculating the bunching factor of an arbitrarily shaped electron beam with a length longitudinal $\Lambda_{b}$ located at a longitudinal distance $d$ from an observer. $\theta$ is the observation angle or the emission angle with respect to the beam trajectory, and $\psi$ is the azimuthal angle in the $x-y$ plane.

The quadratic phase term $\exp \left[i \pi f\left(x_{j}^{\prime 2}+y_{j}^{\prime 2}\right) / c d\right]$ is only contributed by the transverse spread of the particles. If the following condition,

$$
\left\langle\frac{\pi f\left(x_{j}^{\prime 2}+y_{j}^{\prime 2}\right)}{c d}\right\rangle=\frac{\pi f \sigma_{r}^{2}}{c d} \ll 2 \pi,
$$

is satisfied, where $\sigma_{r}$ represents the rms beam radius and \langle\rangle denotes the average over all the electrons of the beam, then the quadratic phase term under the summation sign in Eq. (5) is approximately unity over the entire beam. For example, to satisfy Eq. (6), the observation distance $d$ has to be much larger than $13.3 \mathrm{~cm}$ for our following case with $f=2 \mathrm{PHz}(\lambda=150 \mathrm{~nm})$ and $\sigma_{r}=200 \mu \mathrm{m}$. This far-field condition can be easily achieved for most applications. Under this far-field condition, the bunching factor can be written as

$$
\begin{aligned}
M_{b}(\vec{k}) & =M_{b}\left(k_{x}, k_{y}, k_{z}\right) \\
& \cong \frac{1}{N}\left|\sum_{j=1}^{N} \exp \left(-i k_{z} z_{j}^{\prime}-i k_{x} x_{j}^{\prime}-i k_{y} y_{j}^{\prime}\right)\right| \\
& =\frac{1}{N}\left|\sum_{j=1}^{N} \exp \left(-i \vec{k} \cdot \overrightarrow{R_{j}^{\prime}}\right)\right|,
\end{aligned}
$$

where $k_{x} \cong 2 \pi f x / c d, \quad k_{y} \cong 2 \pi f y / c d, \quad k_{z} \cong 2 \pi f / c$ and $\vec{k}=\left(k_{x}, k_{y}, k_{z}\right)$. The bunching factor written in the form of Eq. (7) depends on the direction and amplitude of $\vec{k}$, which are related to the radiation frequency $f$ and the position of the observer $\vec{R}=(x, y, d)$. Equation (7) is the sum of all the plane-wave-like fields with the wave vector $\vec{k}$ emitted from the sources, which are the electrons in this study. This is a consequence of the far-field approximation made in Eq. (6).

For a beam with a normalized 3D distribution function $n\left(\overrightarrow{R^{\prime}}\right), M_{b}(\vec{k})$ can similarly be expressed as

$M_{b}(\vec{k})=\left|\int_{V} n\left(\overrightarrow{R^{\prime}}\right) \exp \left(-i \vec{k} \cdot \overrightarrow{R^{\prime}}\right) d \overrightarrow{R^{\prime}}\right|=F\left\{n\left(\overrightarrow{R^{\prime}}\right)\right\}$,

where $F\left\{n\left(\overrightarrow{R^{\prime}}\right)\right\}$ is the Fourier transform of $n\left(\overrightarrow{R^{\prime}}\right)$.
Qualitatively, the bunching factor can be regarded as the interference of waves emitted by the electrons in the beam with a normalized amplitude. Considering all the electrons as independent radiation sources, the relationship between the distribution and bunching factor of electrons is analogous to the one between the distribution and interference of point light sources in Fourier optics [44]. Similarly, Eqs. (6) and (8) are analogous to the Fraunhofer approximation and the Fraunhofer diffraction equation in scalar wave diffraction theory [44], respectively.

\section{B. Bunching factor of an electron beam with a small beam radius}

Under the assumptions that the radius, divergence, and energy spread of the electron beam are negligibly small so that $-k_{x} x_{j}^{\prime}-k_{y} y_{j}^{\prime} \ll 2 \pi$, the bunching factors in Eqs. (7) and (8) reduce to the one-dimensional (1D) expressions, called hereafter the longitudinal bunching factors

$$
M_{b, l}(f) \equiv \frac{1}{N}\left|\sum_{j=1}^{N} \exp \left(-i \frac{2 \pi f z_{j}^{\prime}}{c}\right)\right|
$$

and

$M_{b, l}(f) \equiv\left|\int_{-\infty}^{\infty} n\left(z^{\prime}\right) \exp \left(-i \frac{2 \pi f z^{\prime}}{c}\right) d z^{\prime}\right|=\left|\mathbb{F}\left\{n\left(z^{\prime}\right)\right\}\right|$,

respectively. $n\left(z^{\prime}\right)$ is the normalized longitudinal distribution function of the beam, and $\mathbb{F}\left\{n\left(z^{\prime}\right)\right\} \equiv$ $\int_{-\infty}^{\infty} n\left(z^{\prime}\right) \exp \left(-i 2 \pi f z^{\prime} / c\right) d z^{\prime}$ is the Fourier transform of $n\left(z^{\prime}\right)$.

According to Eqs. (9) and (10), the bunching spectrum of an electron beam with a periodical longitudinal modulation at a repetition rate $f$ is expected to have regular peaks at $f$ and its harmonics. The value of the bunching factor is crucially influenced by the quality of this density modulation, including the periodicity and contrast of the microbunches. For example, the bunching factor for a set of 50attosecond (rms) Gaussian microbunches with a repetition rate of $10 \mathrm{PHz}(\lambda=30 \mathrm{~nm})$ is about 0.01 at $f=10 \mathrm{PHz}$. With the effect of the spatial transformation in rf accelerators, as mentioned in Sec. II, it is expected that both $n\left(z^{\prime}\right)$ and $M_{b}$ of an accelerated beam critically depend on the transverse distribution of electrons at the photocathode.

\section{GENERATION OF A STRUCTURED HOLLOW ELECTRON BEAM}

As mentioned in Sec. II, the study of the spatial transformation of an electron layer with an infinitesimal thickness $\delta z \rightarrow 0$ allows one to understand the impulse response of an accelerator system, which reveals the first-order evolution of the transverse-to-longitudinal distribution of 


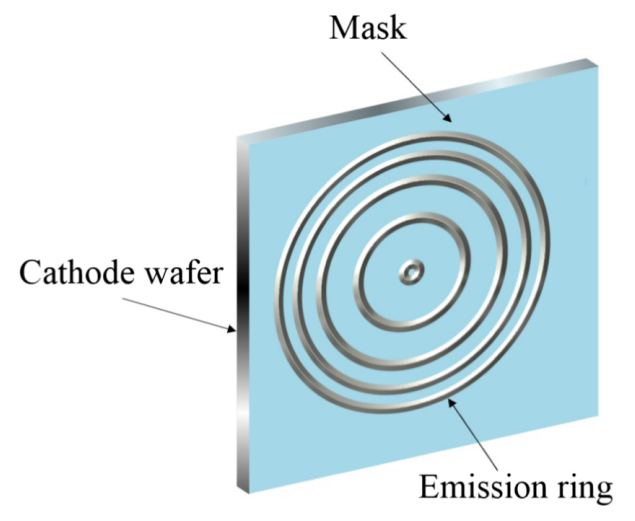

(a)

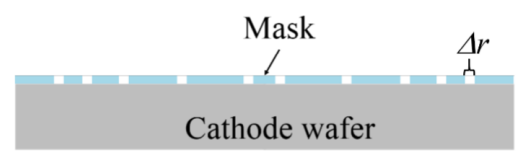

(b)

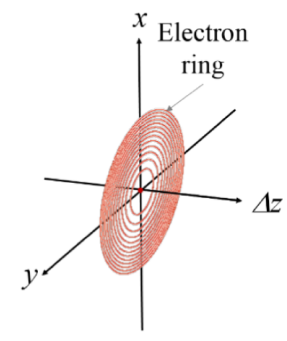

(c)

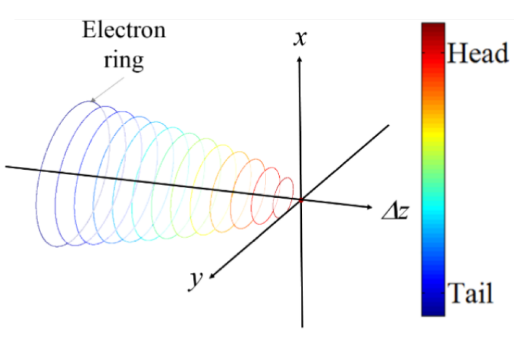

(d)
FIG. 4. (a) Illustration of a multiring structured photocathode. The pattern of the uncovered cathode surface is in the form of concentric rings. (b) Cross section view of the structured photocathode. The cathode material uncovered by the mask emits electrons. (c) Spatial distribution of the emitted electrons at the cathode. (d) At the accelerator exit, the concentric electron rings at the cathode are longitudinally separated by the radially dependent rf fields during acceleration.

accelerated electrons in the system through subsequent convolution calculations. The spatial transformation of the electrons in an rf accelerator can convert the transverse density modulation of an input beam into the longitudinal density modulation of an output beam. With a specified transverse-to-longitudinal conversion of an electron distribution, it is possible to tailor design a transverse electron density distribution at the photocathode for a longitudinal modulation desired at the exit of an accelerator system. This is the scheme that we propose to generate a beam with a PHz longitudinal modulation in an electron beam. In the following, we will now present it in more details.

\section{A. Multiring structured photocathode}

To keep the electron beam cylindrically symmetric during acceleration, we choose to imprint a ring-pattern density modulation onto the beam emitted from a multiring photocathode. Since the electron flux of photoemission is related to the cathode material and the intensity of the driver laser, the transverse distribution of the emitted electrons can be modulated by varying the cathode material or the intensity profile of the driver laser on the photocathode. Figure 4 illustrates a cylindrically symmetric, densitymodulated electron beam generated from a multiring structured photocathode. The multiring structured cathode, shown in Fig. 4(a), can be fabricated by patterning a mask atop a cathode wafer with lithographic techniques $[45,46]$. The cathode wafer is partially covered by the mask, and the pattern of the uncovered cathode area is composed of many concentric rings with an equal linewidth $\Delta r$. The cross section of such a structured photocathode is shown in Fig. 4(b). The photon energy of the driver laser should then be larger than the work function of the cathode ring $\Phi_{\text {ring }}$ but smaller than the one of the mask $\Phi_{\text {mask }}$. In this way, the uncovered cathode rings will emit electron rings of the same pattern when illuminated by the driver laser. For example, the materials of the cathode wafer and mask can be copper and nickel with work functions of 4.31 and $5.01 \mathrm{eV}$, respectively. A 266-nm UV laser with a photon energy of $4.66 \mathrm{eV}$ can be a suitable driver laser to induce photoemission from the copper rings. The lithographically patterned cathode surface is assumed to be smooth enough so as not to induce nonuniform photoemission and emittance growth [47]. Figure 4(c) illustrates the spatial distribution of the electron rings at the cathode. After being

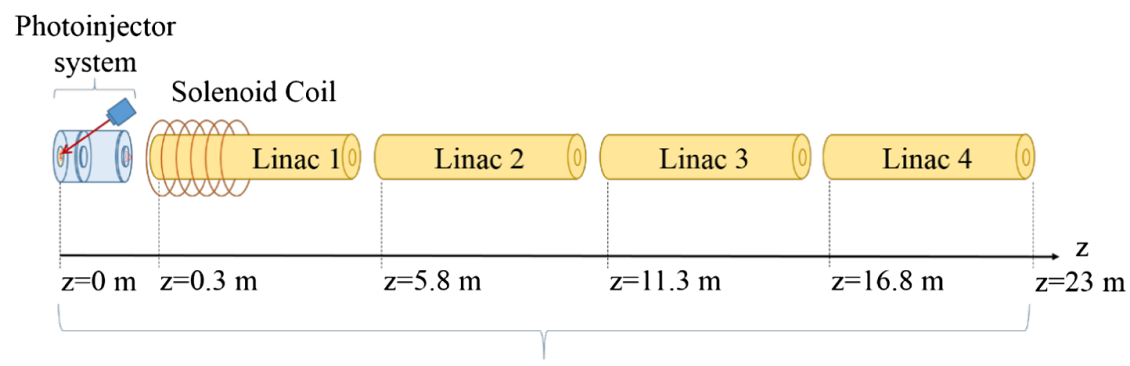

Total length $\sim 23 \mathrm{~m}$

FIG. 5. The $23 \mathrm{~m}$ long NCRF accelerator system considered in our simulation study. 
TABLE I. Parameters of the NCRF accelerator system in Fig. 5.

\begin{tabular}{lc}
\hline \hline Parameter & Value \\
\hline $\begin{array}{l}\text { rms laser pulse duration } \\
\text { Linewidth of each emission } \\
\text { ring on the photocathode }\end{array}$ & $30 \mathrm{fs}$ \\
$\begin{array}{l}\text { Number of emission rings } \\
\text { on the photocathode }\end{array}$ & $30 \mathrm{~nm}$ \\
Photoinjector & 131 \\
& $2.856 \mathrm{GHz}, 1.6$ cell, \\
Injection phase & standing wave \\
Linac 1-4 & $180^{\circ}$ \\
& $2.856 \mathrm{GHz}, 147$ cell, \\
Acceleration gradient & traveling wave \\
$25 \mathrm{MV} / \mathrm{m}$ (Linac 1-4) & $100 \mathrm{MV} / \mathrm{m} \mathrm{(photoinjector)}$ \\
Accelerator length & \\
$5.15 \mathrm{~m}$ (Linac 1-4) & $0.126 \mathrm{~m}(\mathrm{photoinjector})$ \\
Peak B-field in the coil & $7.5 \times 10^{-2} \mathrm{~T}$ \\
Coil length & $2 \mathrm{~m}$ \\
\hline \hline
\end{tabular}

"In ASTRA, we set Nrad $=10$, Nlong_in $=30$ for the cylindrical grid when performing the space charge calculation [39].

accelerated by the radially dependent rf fields, the electron rings with different radii are separated and transformed into a longitudinally density-modulated hollow beam, as illustrated conceptually in Fig. 4(d).

After the spatial transformation during acceleration, the lengths of the microbunches are related to both the thickness of the particle layer $\delta z$ and the linewidth of the input electron rings $\Delta r$ because of the radial induced curvature shown in Fig. 2(a). With today's electron beam lithography or extreme ultraviolet (EUV) photolithography, the achievable feature size of the structured cathode can be on the order of 10-30 nm [46]. To be conservative, we assume $\Delta r=30 \mathrm{~nm}$ thereafter. The lengths of the output electron rings are mainly determined by the laser pulse duration, beam charge, peak acceleration gradient and injection phase in the rf field.

\section{B. PHz structured hollow electron beam}

In this section, we present our study using the simulation code ASTRA for the generation of a hollow structured electron beam with a longitudinal bunching frequency of $2 \mathrm{PHz}$ from a 23-m long NCRF accelerator system. The accelerator system is composed of a 1.6-cell photoinjector with a multiring structured cathode and four downstream linear accelerator (linac) sections, as illustrated in Fig. 5. The first section is partially wrapped with solenoid coils, which effectively compensate the emittance growth and provide transverse focusing to the beam [48]. The conceived 23-m NCRF accelerator system is just an illustration and can be further optimized for real experiments. Table I lists the design parameters of the NCRF accelerator system considered in our simulation.

Once the electron beam is accelerated to a relativistic velocity by the four linac sections, the relative longitudinal position of the electrons beyond $z=23 \mathrm{~m}$ is almost fixed, implying an almost invariant longitudinal modulation. The beam is transversely focused at $z \sim 55 \mathrm{~m}$ by the solenoid coils. The rms beam radius along the beam line is shown in Fig. 6(a), kept under $200 \mu$ min the focal region between $z=45$ and $65 \mathrm{~m}$. The $20-\mathrm{m}$ depth of focusing is convenient for installing, for instance, an undulator to generate radiation. The position and length of the focal region are correlated, and can be adjusted by varying the magnetic field of the solenoid coils. For example, by adjusting the magnetic field of the solenoid coils between 0.07 and $0.08 \mathrm{~T}$, the position of the focal region of the 100-fC electron beam can be varied between 95 and $34 \mathrm{~m}$, and the depth of focus can be varied between 38 and $15 \mathrm{~m}$. It is certainly possible to use the solenoid coils primarily for emittance compensation and install a few quadrupole lenses after the accelerator system to focus the beam at a desired location. For this study, the solenoid coils are sufficient to serve our purposes.

During acceleration, the transverse distribution of a lowcharge beam is mainly influenced by the radial component

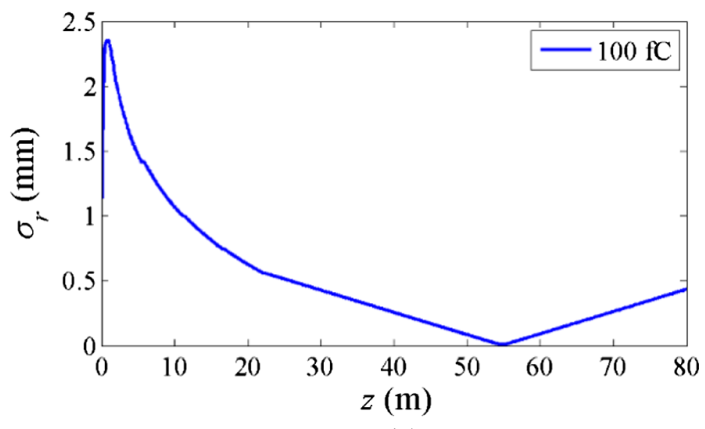

(a)

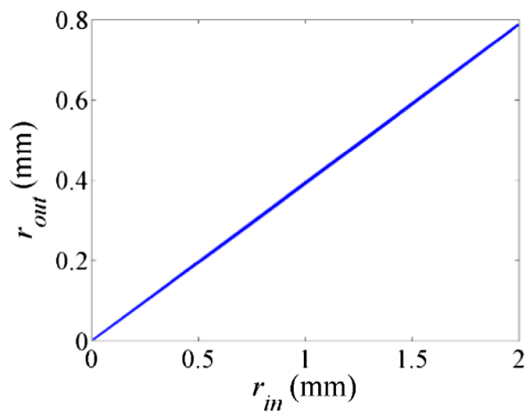

(b)

FIG. 6. (a) rms beam radius $\sigma_{r}$ as a function of the longitudinal position $z$ for a 100-fC beam in the proposed 23-m beam line in Fig. 5 . The solenoid coils focus the beam at $z \sim 55 \mathrm{~m}$. The rms beam radius is kept under $200 \mu \mathrm{m}$ between $z=45$ to $65 \mathrm{~m}$ for, say, a radiation insertion device. (b) Linear relationship found from simulation between the input and output radial positions $r_{\text {in }}$ (at $z=0 \mathrm{~m}$ ) and $r_{\text {out }}$ (at $z=23 \mathrm{~m}$, the position of the accelerator exit) of the electrons for the $100-\mathrm{fC}$ beam. 


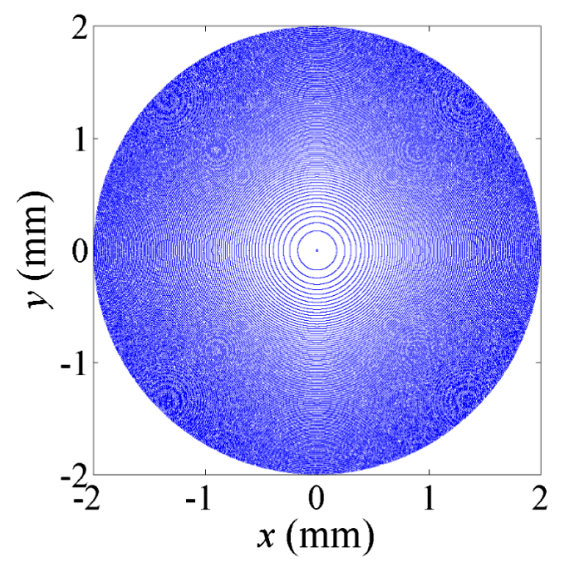

FIG. 7. Transverse distribution of the input electron beam at the cathode. In this design, the number of electron rings is 131 and the linewidth of each electron ring is set to $30 \mathrm{~nm}$. The chosen dimensions are within the reach of today's nanofabrication techniques.

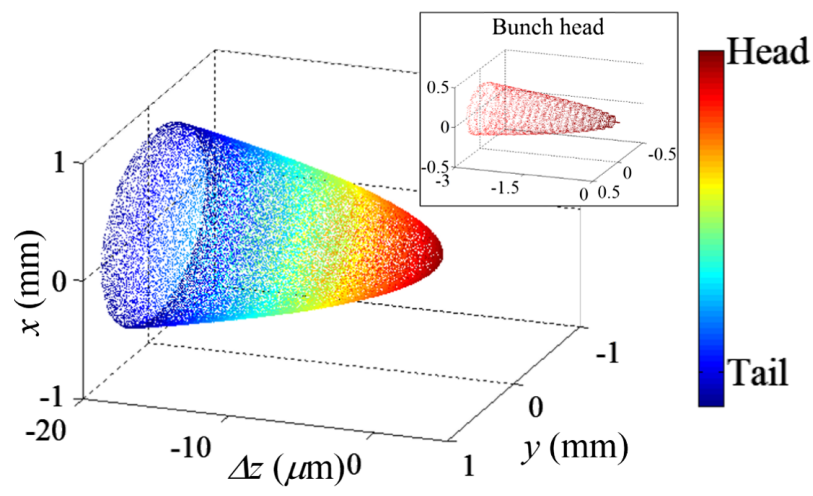

(a)

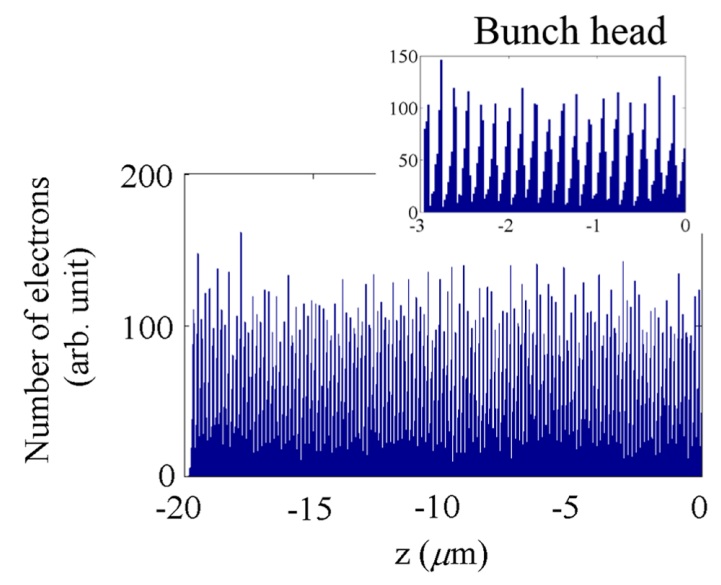

(c) of the electric field in the rf cavities and by the magnetic field of the solenoid coils. In the paraxial region, the expansion of the field $E_{r 0}(r) \propto J_{1}\left(\eta_{0} r\right)=\eta_{0} r / 2-\left(\eta_{0} r\right)^{3} /$ $16+\cdots \approx \eta_{0} r / 2$ is kept up to the linear term. Therefore, the radial electric force experienced by an electron is approximately proportional to its radial position in the rf cavity. Since the solenoid coils transversely confine the beam with a constant magnification [49] and the electron transit time is approximately a constant for all electrons in the beam through the accelerator system, the radial displacement of the electrons is more or less proportional to $r$ in the paraxial region when the space charge effects are negligible. Indeed, Fig. 6(b) shows a linear relationship or a constant slope $M \equiv d r_{\text {out }} / d r_{\text {in }}$ between the radial positions $r_{\text {in }}($ at $z=0 \mathrm{~m})$ and $r_{\text {out }}($ at $z=23 \mathrm{~m}$ ) of the electrons. As it will be shown below, this relation is crucial for the design of the structured photocathode.

In our simulation, the transverse distribution of the input beam is optimized for a goal to generate $2 \mathrm{PHz}$ repetitive microbunches at $z=23 \mathrm{~m}$. Figure 7 shows such a designed

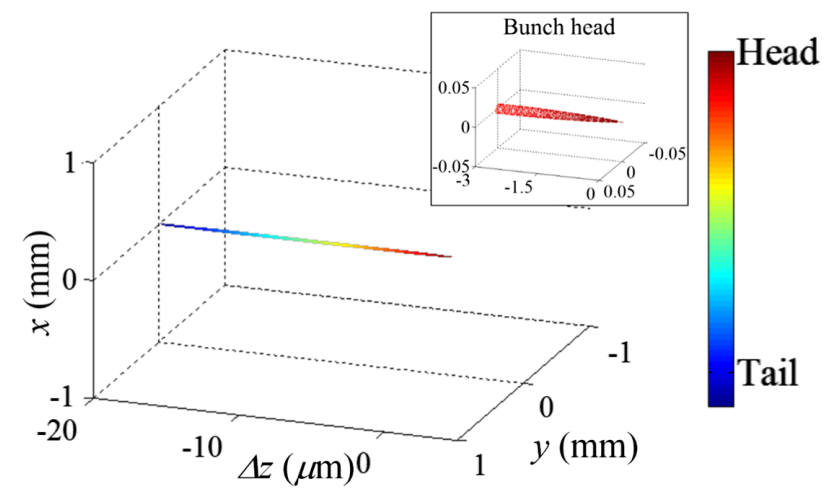

(b)

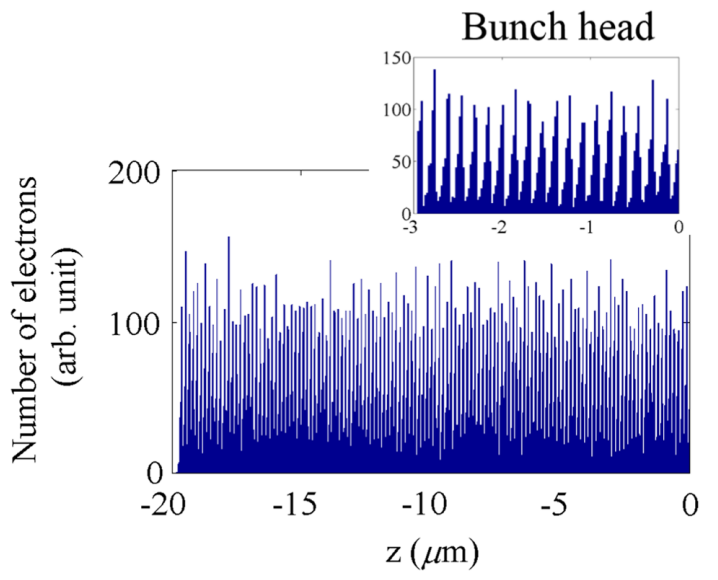

(d)

FIG. 8. Spatial distributions of the hollow structured beam at the (a) exit of the rf accelerator system $(z=23 \mathrm{~m})$ and (b) focal point of the electron beam $(z=55 \mathrm{~m})$. Longitudinal distributions of the beam at $(\mathrm{c}) z=23 \mathrm{~m}$ and $(\mathrm{d}) z=55 \mathrm{~m}$. The subplot show the zoom-in views near the bunch head. 
transverse electron distribution at the cathode. There are 131 electron rings within a circular radius of $2 \mathrm{~mm}$. The radial space between two successive rings is in the range of a few $\mu \mathrm{m}$ to a few tens of $\mu \mathrm{m}$. The initial ring radii are crucial to fine-tune the longitudinal distances between the rings and hence the bunching frequency of the output beam after acceleration. The linewidth of each ring is set to $30 \mathrm{~nm}$ and the total charge is set to $100 \mathrm{fC}$ in the simulation. Both the rms pulse duration of the driver laser and the rms temporal length of the electron rings at the cathode are set to $30 \mathrm{fs}$. The radii of the rings are calculated according to a known $r-\Delta z$ distribution of an electron layer at the exit of the accelerator system. The charges of all the electron rings are assumed to be approximately the same. We randomly generate electrons on the rings with equal probability to create the input particle files for our simulations. The proposed charge distribution can be obtained by generating a laser intensity inversely proportional $r$ in a properly programmed spatial light modulator. This charge manipulation is chosen for creating the input particle files conveniently and demonstrating the electron bunching in the downstream beam line. Further optimization for, say, minimizing emittance growth remains open in future studies.

A self-developed algorithm is used to calculate the radii of the cathode rings and generate a corresponding input particle file in the simulation. Specifically, we first simulate the propagation of a thin electron layer in the accelerator system to generate a $r-\Delta z$ distribution of particles similar to the one in Fig. 2 and a $r_{\text {out }}-r_{\text {in }}$ distribution similar to the one in Fig. 6(b) at the accelerator exit. We then sliced the cone of the $r-\Delta z$ distribution for a given longitudinal period in $z$ to obtain a set of rings similar to those in Fig. 4(d). The linear relationship between $r_{\text {in }}$ and $r_{\text {out }}$ of the electrons allows us to divide the radii of the sliced rings by a constant ratio $M$ to obtain the radii of the rings at the photocathode. We ignored the space charge when calculating the cathode rings, but included it in the simulations.

Figures 8(a) and 8(b) show the 3D spatial distributions of the output beam at the accelerator exit $z=23 \mathrm{~m}$ and at the focal point $z=55 \mathrm{~m}$, respectively. The insets of Figs. 8(a) and 8(b) are the zoom-in views of the bunch heads at $z=$ 23 and $55 \mathrm{~m}$, respectively, where longitudinally separated electron rings are clearly seen. Figures 8(c) and 8(d) show the longitudinal distributions of the beam or histograms of the particles at $z=23$ and $55 \mathrm{~m}$, respectively, and the insets show the zoom-in views at the bunch head. Periodic bunching of the output beam is clearly seen from the plots. Table II summarizes the relevant parameters of the output beam. In our simulation, the number of particles, 625,000 , is matched to the exact number of the electrons in the 100-fC bunch charge. Therefore, the space charge field and electron behavior can be accurately calculated by the simulation code to reflect a real electron beam in the accelerator system.
TABLE II. Parameters of the output electron beam.

\begin{tabular}{lc}
\hline \hline Parameter & Value \\
\hline Average energy & $392.5 \mathrm{MeV}$ \\
Total charge & $100 \mathrm{fC}$ \\
rms macrobunch length & $5.69 \mu \mathrm{m}$ \\
rms macrobunch duration & $18.97 \mathrm{fs}$ \\
Full macrobunch length & $\sim 20 \mu \mathrm{m}$ \\
Full macrobunch duration & $66.67 \mathrm{fs}$ \\
rms beam current & $5.27 \mathrm{~A}$ \\
Average charge per microbunch & $0.76 \mathrm{fC}$ \\
rms beam divergence & $<18.6 \mu \mathrm{rad}$ \\
Transverse emittance (normalized) & $0.04 \pi \mathrm{mm} \mathrm{mrad}$. \\
Repetition rate of microbunches & $2 \mathrm{PHz}$ \\
Number of particles in the simulation & 625,000 \\
\hline \hline
\end{tabular}

Figure 9(a) shows the longitudinal bunching spectrum of the 100-fC beam from our simulation at $z=23 \mathrm{~m}$ (blue curve), calculated by using Eq. (10). As a comparison, we also plot in the same figure the bunching spectrum for a 500-fC output beam (red curve). Figure 9(b) shows the longitudinal distribution of a structured hollow beam with a

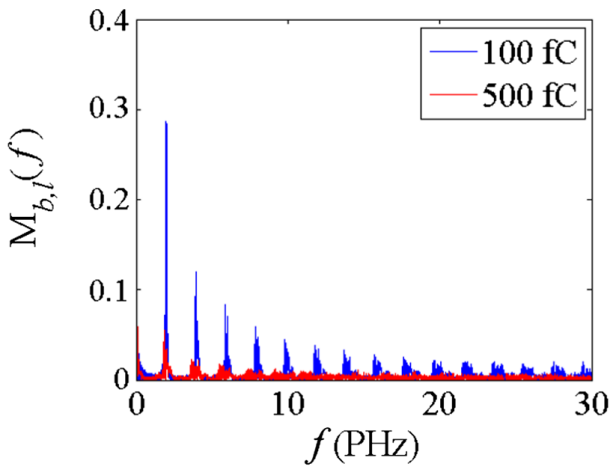

(a)

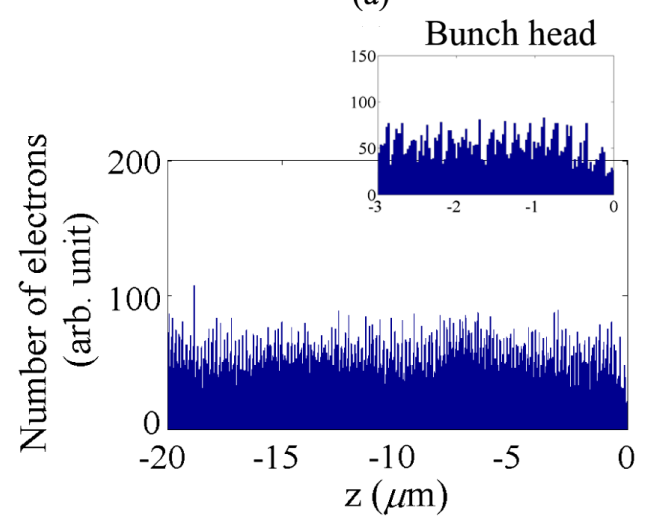

(b)

FIG. 9. (a) Longitudinal bunching spectra of simulated output beams with total charges of 100 and $500 \mathrm{fC}$ at $z=23 \mathrm{~m}$. (b) Longitudinal distribution of a 500-fC structured hollow beam at $z=23 \mathrm{~m}$. Space charge fields in the $500-\mathrm{fC}$ beam degrade the bunching quality. The subplot shows nevertheless a visible periodical modulation on the electron density at the bunch head. 
total charge of $500 \mathrm{fC}$ at $z=23 \mathrm{~m}$. Comparing Fig. 9(b) with Fig. 8(c), one can see that the contrast of the microbunches decreases when the total charge of the beam increases from 100 to $500 \mathrm{fC}$. The bunching spectrum of the 100-fC beam peaks at the harmonics of $2 \mathrm{PHz}$ $(\lambda=150 \mathrm{~nm})$, and retains a value of 0.02 at the 15 th harmonic near $30 \mathrm{PHz}(\lambda=10 \mathrm{~nm})$. The bunching factor of the 500-fC beam attains a value about 0.05 and 0.02 at $f=2$ and $4 \mathrm{PHz}(\lambda=150$ and $75 \mathrm{~nm})$, respectively, although the space charge effects in this higher charge beam tend to smear out the electron bunches and therefore degrade the modulation quality. The simulation results demonstrate that, by manipulating the transverse electron distribution at the photocathode, it is possible to generate a longitudinally density-modulated relativistic electron beam with a high bunching factor up to few tens of $\mathrm{PHz}$ after acceleration in the considered accelerator system.

\section{Comparison between bunched hollow and solid electron beams}

Equation (3) indicates that the coherent radiation energy is strongly influenced by the bunching factor of an electron beam. A longitudinal density modulation at $f$ in a beam increases the bunching factor and can therefore greatly increase the coherent radiation emitted at $f$ in a superradiant emission process. Previously, we calculated the bunching spectrum of the simulated structured beam by ignoring the transverse extent of the beam. In this section, we theoretically take into account the transverse profile of the beam and analyze the so-called 3D bunching factor for our case in comparison with a longitudinally modulated solid beam.

For an electron beam with a distribution function $n\left(\overrightarrow{R^{\prime}}\right)=n\left(r^{\prime}, z^{\prime}\right)$, where $r^{\prime} \equiv \sqrt{x^{\prime 2}+y^{\prime 2}}$, the phase $\vec{k} \cdot \vec{R}^{\prime}$ in Eqs. (7) and (8) can be written in the form

$\vec{k} \cdot \overrightarrow{R^{\prime}}=k_{x} x^{\prime}+k_{y} y^{\prime}+k_{z} z^{\prime}=k_{r} r^{\prime} \cos \left(\varphi^{\prime}-\psi\right)+k_{z} z^{\prime}$,

where $k_{r}=\sqrt{{k_{x}}^{2}+k_{y}^{2}} \cong 2 \pi f \theta / c$ is the radial component of the wave number, $\psi=\tan ^{-1}\left(k_{y} / k_{x}\right)=\tan ^{-1}(y / x)$ and $\varphi^{\prime}=\tan ^{-1}\left(y^{\prime} / x^{\prime}\right)$ are the azimuthal angles in the coordinates of the observer and the electron, respectively. Equation (11) is particularly useful for a system with cylindrical symmetry, as it is often the case for an electron-beam system. With the help of Eq. (11), one can rewrite the volume integral in Eq. (8) in the cylindrical coordinate system [43], and obtain the bunching factor of a cylindrically symmetric electron beam as

$$
\begin{aligned}
M_{b}\left(k_{r}, k_{z}\right) & =\left|\int_{-\Lambda_{b}}^{0} \int_{0}^{\infty} \int_{0}^{2 \pi} n\left(r^{\prime}, z^{\prime}\right) \exp \left\{-i\left[k_{r} r^{\prime} \cos \left(\varphi^{\prime}-\psi\right)+k_{z} z^{\prime}\right]\right\} r^{\prime} d \varphi^{\prime} d r^{\prime} d z^{\prime}\right| \\
& =\left|2 \pi \int_{-\Lambda_{b}}^{0} \int_{0}^{\infty} n\left(r^{\prime}, z^{\prime}\right) J_{0}\left(k_{r} r^{\prime}\right) r^{\prime} d r^{\prime} \exp \left(-i k_{z} z^{\prime}\right) d z^{\prime}\right|,
\end{aligned}
$$

where the relation $J_{0}(x)=\frac{1}{2 \pi} \int_{-\pi}^{\pi} \exp [i(-x \sin t)] d t$ has been used in the derivation of Eq. (12), and the cylindrical symmetry of the beam makes $M b$ independent of $\varphi^{\prime}$ and $\psi$.

As an example, we consider a longitudinally densitymodulated solid beam with a uniform transverse distribution within a cutoff radius $r_{0}$, and assume that all the microbunches constituting the beam are infinitely short and periodically spaced by a distance $c / f_{b}$ along the longitudinal direction. The 3D distribution function of such a flattop beam can be expressed as

$$
n_{\text {flattop }}\left(r^{\prime}, z^{\prime}\right)=\frac{1}{N_{m}} \sum_{j=1}^{N_{m}} \frac{\Pi\left(r^{\prime}-r_{0}\right)}{\pi r_{0}^{2}} \delta\left(z^{\prime}-z_{j}\right),
$$

where $z_{j}=(1-j) c / f_{b}$ is the longitudinal position of the $j$ th electron microbunch, $N_{m}$ is the total number of microbunches, and $\Pi\left(r^{\prime}-r_{0}\right)$ is a step function with $\Pi\left(r^{\prime}-r_{0}\right)=1$ for $r^{\prime}-r_{0} \leq 0$ and $\Pi\left(r^{\prime}-r_{0}\right)=0$ elsewhere. By substituting this distribution function into Eq. (12), the bunching factor can be calculated as

$$
\begin{aligned}
M_{b, \text { flattop }}\left(k_{r}, k_{z}\right) & =\left|\frac{2}{N_{m} r_{0}^{2}} \sum_{j=1}^{N_{m}} \int_{-\Lambda_{b}}^{0} \int_{0}^{r_{0}} J_{0}\left(k_{r} r^{\prime}\right) r^{\prime} d r^{\prime} \delta\left(z^{\prime}-z_{j}\right) \exp \left(-i k_{z} z^{\prime}\right) d z^{\prime}\right| \\
& =\left|\frac{2}{N_{m} k_{r} r_{0}} J_{1}\left(k_{r} r_{0}\right) \sum_{j=1}^{N_{m}} \exp \left(-i k_{z} z_{j}\right)\right|
\end{aligned}
$$


According to Eq. (14), the transverse coherence of the radiation is related to $k_{r} r_{0}$. For relativistic electrons, the typical emission angle $\theta$ of synchrotron radiation is within the value of the inverse Lorentz factor $\gamma^{-1}$ [43]. The condition for full transverse coherence is $k_{r} r_{0}=2 \pi f r_{0} \theta /$ $c \approx 2 \pi f r_{0} / c \gamma \ll 1$, so that $J_{1}\left(k_{r} r_{0}\right) /\left(k_{r} r_{0}\right)$ retains its maximum value at $k_{r} r_{0}=0$ [43].
Considering now a hollow structured beam composed of a set of electron rings with an equal linewidth $\Delta r$ at different radii, and assuming that the electron rings are infinitely short and periodically spaced by $c / f_{b}$ along the longitudinal direction, one can write the $3 \mathrm{D}$ distribution function of such a hollow structured beam as

$$
n_{\text {hollow }}\left(r^{\prime}, z^{\prime}\right)=\frac{1}{N_{m}} \sum_{j=1}^{N_{m}} \frac{1}{2 \pi r_{j} \Delta r}\left[\Pi\left(r^{\prime}-r_{j}-\frac{\Delta r}{2}\right)-\Pi\left(r^{\prime}-r_{j}+\frac{\Delta r}{2}\right)\right] \delta\left(z^{\prime}-z_{j}\right) .
$$

where $r_{j}$ is the radius of the $j$ th ring. By substituting the distribution function into Eq. (12), one can obtain the bunching factor of the hollow structured beam as

$$
M_{b, \text { hollow }}\left(k_{r}, k_{z}\right)=\left|\frac{1}{N_{m}} \sum_{j=1}^{N_{m}} \frac{1}{r_{j} \Delta r k_{r}}\left\{\left(r_{j}+\frac{\Delta r}{2}\right) J_{1}\left[k_{r}\left(r_{j}+\frac{\Delta r}{2}\right)\right]-\left(r_{j}-\frac{\Delta r}{2}\right) J_{1}\left[k_{r}\left(r_{j}-\frac{\Delta r}{2}\right)\right]\right\} \exp \left(-i k_{z} z_{j}\right)\right| .
$$

If $\Delta r \rightarrow 0$, i.e., $\Delta r \ll r_{j}$ for any $j$, then Eqs. (15) and (16) reduce to

$$
n_{\text {hollow }}\left(r^{\prime}, z^{\prime}\right)=\frac{1}{N_{m}} \sum_{j=1}^{N_{m}} \frac{1}{2 \pi r_{j}} \delta\left(r^{\prime}-r_{j}\right) \delta\left(z^{\prime}-z_{j}\right) .
$$

and

$M_{b, \text { hollow }}\left(k_{r}, k_{z}\right)=\left|\frac{1}{N_{m}} \sum_{j=1}^{N_{m}} J_{0}\left(k_{r} r_{j}\right) \exp \left(-i k_{z} z_{j}\right)\right|$,

respectively. Equation (18) indicates that the condition for full transverse coherence for the radiation emitted from a structured hollow beam is $2 \pi f r_{0} / c \gamma \ll 1$ [43], since the function $J_{0}\left(k_{r} r_{0}\right)$ is maximal at $k_{r} r_{0}=0$ with $r_{0}$ being the radius of the outermost ring. This condition is similar to that for a longitudinally modulated solid beam.

At $f=f_{b}$, Eqs. (14) and (18) become

$$
\begin{aligned}
M_{b, \text { flattop }}\left(k_{r}, k_{z}\right) & =M_{b, \text { flattop }}\left(\theta, f_{b}\right) \\
& =\left|\frac{c}{\pi f_{b} \theta r_{0}} J_{1}\left(\frac{2 \pi f_{b} \theta r_{0}}{c}\right)\right|
\end{aligned}
$$

and

$$
\begin{aligned}
M_{b, \text { hollow }}\left(k_{r}, k_{z}\right) & =M_{b, \text { hollow }}\left(\theta, f_{b}\right) \\
& =\left|\frac{1}{N_{m}} \sum_{j=1}^{N_{m}} J_{0}\left(\frac{2 \pi f_{b} \theta r_{j}}{c}\right)\right|,
\end{aligned}
$$

respectively. Since the longitudinal bunching factors of these two beams are unity at $f=f_{b}$, Eqs. (19) and (20) can be regarded as the transverse bunching factors of the solid and hollow beams, respectively. The transverse bunching factor of a beam is related to the transverse beam distribution and observation angle $\theta$. In Fig. 10, we compare the angular spectra of the transverse bunching factors of the solid beams (blue curves) and hollow beams from our simulation (red curves) with cutoff beam radii at

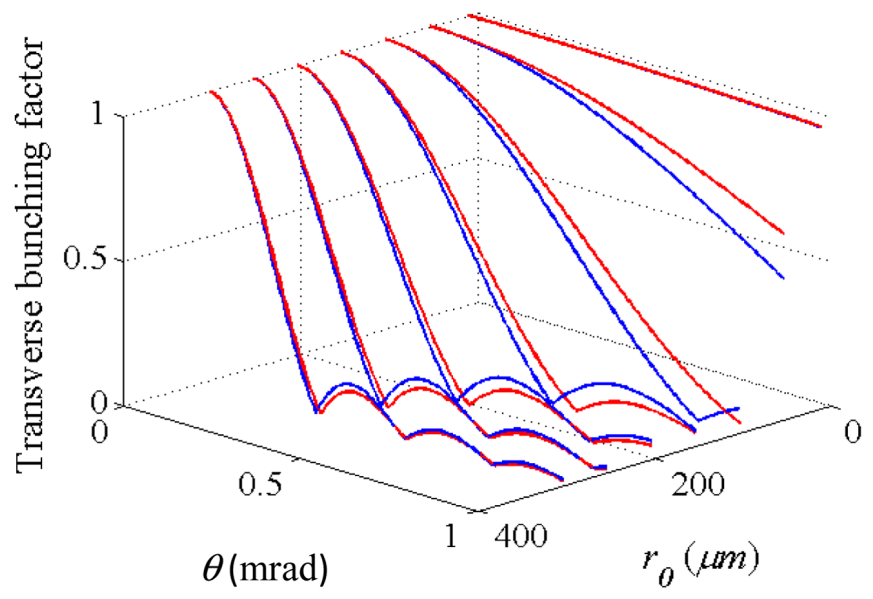

FIG. 10. Angular spectra of the transverse bunching factors of the hollow (red curves) and the solid (blue curves) beams with $r_{0}=10,50,100,150,200,250$ and $300 \mu \mathrm{m}$. With the solenoid field in our beam line, the beam radius varies along $z$. In this plot, the particle distributions of the hollow beam with $r_{0}=300,250$, 200, 150, 100, 50 and $10 \mu \mathrm{m}$ are obtained at $z=50.88,48.96$, $47.04,45.12,43.20,41.28,39.36 \mathrm{~m}$, respectively, from the simulation of the considered beam line. 
$r_{0}=10,50,100,150,200,250$ and $300 \mu \mathrm{m}$. In Fig. 10, one can see that the transverse bunching factors of the two beams decrease with the increase of the observation angle $\theta$ and cutoff beam radius $r_{0}$. From the angular spectra of the transverse bunching factors, one can record a pattern in the observation plane (the $x-y$ plane in Fig. 3) containing concentric rings around the central maximum at $\theta=0^{\circ}$. This pattern results from the superposition of the radiation fields of the individual electrons in a circular beam. When the beam radius $\sigma_{r}$ and observation angle $\theta$ are negligibly small such that Eq. (6) and the condition for fully transverse coherence are satisfied, the transverse bunching factors of the hollow and solid beams coincide with each other, approaching a magnitude of unity. Similar to Eqs. (7) and (8) reducing to Eqs. (9) and (10) in the paraxial region, the 3D bunching factors of the solid and hollow beams also reduce to the $1 \mathrm{D}$ bunching factor in the paraxial limit. As Fig. 10 shows, in the region $\theta \ll 1$, the blue and red curves are well overlapped. Consequently, in the paraxial limit, a density-modulated hollow beam is equally useful as a density-modulated solid beam for generating coherent radiation in the far-field zone.

\section{CONCLUSION}

In this paper, we present a novel scheme to generate high-frequency periodic bunches within an electron beam. Illuminating a multiring structured photocathode with a laser pulse, as illustrated by Fig. 4, allows one to precisely modulate the transverse density of an input beam. This input beam is further transformed into a longitudinally density modulated output beam by the radially dependent rf fields in an rf photoinjector and subsequent accelerator sections. Figure 5 exemplifies such an accelerator system. Our computer simulation shows that the transverse density modulation of a $100-\mathrm{fC}$ input electron beam at a multiring photocathode of an S-band photoinjector can be transformed into longitudinal density modulation at $\mathrm{PHz}$ frequencies within a hollow structured beam after acceleration in a 23-m-long NCRF accelerator system. In our design study, the structured photocathode is composed of 131 emission rings with a linewidth of $30 \mathrm{~nm}$ within a $2 \mathrm{~mm}$ radius, as shown in Fig. 7. The modulation frequency of the electron density is primarily determined, and can therefore be adjusted by the radial spacing between the emission rings on the photocathode surface. The design result in Fig. 9(a) shows that the longitudinal bunching spectrum of the output beam reaches 0.3 at $2 \mathrm{PHz}$ and 0.02 at $30 \mathrm{PHz}$. At $30 \mathrm{PHz}$, the radiation wavelength is $10 \mathrm{~nm}$, approaching the soft $\mathrm{x}$-ray spectrum.

The electron emittance, bunch charge, and bunch length in our study are highly similar to those being used for $\mathrm{MeV}$ ultrafast electron diffraction [50]. However, an ordinary photocathode could induce nontrivial thermal emittance to affect the nanobunching in the downstream beam line. Although thermal emittance is not considered in our study, there have been several research efforts and experimental demonstrations to effectively reduce the intrinsic emittance at a photocathode. Those new schemes include transmission-type laser excitation [51] and cryogenic cooling [52]. The space charge force in the electrons also plays an adverse role in the bunching process. In the high-charge regime, when the electron separation is shorter than the Debye length of the electron plasma, the interparticle collisions or the so-called disorder induced heating (DIH) can degrade the emittance [53] and the nanobunching. The simulation code, ASTRA, adopts a mean-field algorithm to calculate the space charge effect and DIH is not considered in our study. However, for DIH to be comparable to the temperature of the photoemission of a cold cathode, the electron number density has to be about $10^{19} \mathrm{~m}^{-3}$. [54] To avoid DIH, we start with a low number density of electrons of about $2 \times 10^{16} \mathrm{~m}^{-3}$ at the photoinjector. Further optimization on beam transport and acceleration is needed to ensure a nontrivial bunching factor in a high-charge beam. Recently, the development of high-gradient THz [55] and optical-frequency accelerators [56] has attracted attention across the community. The miniature laser-powered dielectric undulator [57] is also promising for radiation generation in the x-ray spectrum. To speed up the research and development, there has been a quest for a nanobunched fC-charge beam to inject the advanced accelerator and undulator. The 100-fC nanobunched high-energy beam presented in this study is well suited for this purpose.

Apart from fabricating a nanostructured photocathode surface, one could in principle install a ring-structured mask into an accelerator beam line to introduce similar transverse modulation to an electron beam and generate longitudinally density-modulated electrons at the output. The primary advantage of this alternative is that different masks for different bunching frequencies can be installed to or removed from the beam line with ease. However, to generate a $\mathrm{PHz}$ electron density modulation, the nanorings in the mask might not survive from electron bombardment. Nevertheless, this alternative appears feasible for generating electron density modulation in the $\mathrm{THz}$ regime.

Our theoretical analysis of the 3D bunching factor indicates that the characteristics of radiation, including spectral power and coherence, emitted from a hollow ring and flattop solid beams are nearly indistinguishable in the far-field region and under paraxial approximation, as shown in Fig. 10. When the transverse beam size is very small compared to the observation distance, as quantitatively defined by Eq. (6), our analysis shows that the radiation characteristic of an electron beam in such a paraxial region can be simply described by the 1D longitudinal bunching factor of the beam.

Although we have targeted the bunching frequency in the more difficult $\mathrm{PHz}$ range for valuable EUV and soft x-ray radiations, the feasibility study at $\mathrm{PHz}$ can also open up 
similar opportunities for less challenging, lower frequency radiations. While it is relatively difficult to generate an electron beam with a bunching frequency in the $\mathrm{x}$-ray spectrum, the proposed spatial beam manipulation scheme is useful and relatively simple for generating electron bunches in the THz, EUV, or soft x-ray spectrum. The advantage of the proposed scheme is that particle acceleration and bunching are performed simultaneously in an accelerator system, leaving out the dispersive elements and seed lasers often adopted in the other bunching schemes. It is our next effort to consider the combination of our scheme with others to improve the bunching quality in the hard $\mathrm{x}$-ray spectrum.

\section{ACKNOWLEDGMENTS}

F.-H. C. acknowledges a Ph.D. scholarship support from National Synchrotron Radiation Research Center, Taiwan. Part of this work is supported by Ministry of Science and Technology under Grants No. MOST 108-2112-M-007017-MY3, No. 109-2221-E-007-110, No. 109-2622-E007-021, and No. 109-2923-M-007-006.

[1] A. Gover, Phys. Rev. ST Accel. Beams 8, 030701 (2005).

[2] A. Gover, E. Dyunin, Y. Lurie, Y. Pinhasi, and M. V. Krongauz, Phys. Rev. ST Accel. Beams 8, 030702 (2005).

[3] M. Arbel, A. Gover, A. Eichenbaum, and H. Kleinman, Phys. Rev. ST Accel. Beams 17, 020705 (2014).

[4] P. Zhang, L. K. Ang, and A. Gover, Phys. Rev. ST Accel. Beams 18, 020702 (2015).

[5] D. Yu. Sergeeva, A. A. Tishchenko, and M. N. Strikhanov, Phys. Rev. ST Accel. Beams 18, 052801 (2015).

[6] H. L. Andrews et al., Phys. Rev. ST Accel. Beams 17, 052802 (2014).

[7] J. Breuer, R. Graf, A. Apolonski, and P. Hommelhoff, Phys. Rev. ST Accel. Beams 17, 021301 (2014).

[8] P. Gorham, D. P. Saltzberg, P. Schoessow, W. Gai, J. G. Power, R. Konecny, and M. E. Conde, Phys. Rev. E 62, 8590 (2000).

[9] T. Y. Alekhina and A. V. Tyukhtin, Phys. Rev. ST Accel. Beams 17, 071302 (2014).

[10] T. Aumeyr, M. G. Billing, L. M. Bobb, B. Bolzon, E. Bravin, P. Karataev, K. Kruchinin, T. Lefevre, and S. Mazzoni, Phys. Rev. ST Accel. Beams 18, 042801 (2015).

[11] L. G. Sukhikh, G. Kube, S. Bajt, W. Lauth, Y. A. Popov, and A.P. Potylitsyn, Phys. Rev. ST Accel. Beams 17, 112805 (2014).

[12] I. Nozawa, K. Kan, J. Yang, A. Ogata, T. Kondoh, M. Gohdo, K. Norizawa, H. Kobayashi, H. Shibata, S. Gonda, and Y. Yoshida, Phys. Rev. ST Accel. Beams 17, 072803 (2014).

[13] M. Arbel, A. Abramovich, A. L. Eichenbaum, A. Gover, H. Kleinman, Y. Pinhasi, and I. M. Yakover, Phys. Rev. Lett. 86, 2561 (2001).

[14] R. Bonifacio, L. De Salvo Souza, P. Pierini, and N. Piovella, Nucl. Instrum. Methods Phys. Res., Sect. A 296, 358 (1990).
[15] T. Watanabe, X. J. Wang, J. B. Murphy, J. Rose, Y. Shen, T. Tsang, L. Giannessi, P. Musumeci, and S. Reiche, Phys. Rev. Lett. 98, 034802 (2007).

[16] E. Allaria et al., Nat. Photonics 7, 913 (2013).

[17] L. H. Yu et al., Science 289, 932 (2000).

[18] E. Hemsing, G. Stupakov, D. Xiang, and A. Zholents, Rev. Mod. Phys. 86, 897 (2014).

[19] G. Wang, W. Zhang, G. Wu, D. Dai, X. Yang, C. Feng, M. Zhang, H. Deng, D. Wang, and Z. Zhao, Phys. Rev. ST Accel. Beams 18, 060701 (2015).

[20] E. Hemsing, D. Xiang, M. Dunning, S. Weathersby, C. Hast, and T. Raubenheimer, Phys. Rev. ST Accel. Beams 17, 010703 (2014).

[21] E. Hemsing and D. Xiang, Phys. Rev. ST Accel. Beams 16, 010706 (2013).

[22] M. Dunning, C. Hast, E. Hemsing, K. Jobe, D. McCormick, J. Nelson, T. O. Raubenheimer, K. Soong, Z. Szalata, D. Walz, S. Weathersby, and D. Xiang, Phys. Rev. Lett. 109, 074801 (2012).

[23] D. Xiang, Z. Huang, and G. Stupakov, Phys. Rev. ST Accel. Beams 12, 060701 (2009).

[24] D. Xiang, E. Colby, M. Dunning, S. Gilevich, C. Hast, K. Jobe, D. McCormick, J. Nelson, T. O. Raubenheimer, K. Soong, G. Stupakov, Z. Szalata, D. Walz, S. Weathersby, M. Woodley, and P.-L. Pernet, Phys. Rev. Lett. 105, 114801 (2010).

[25] Z. Wang, D. Huang, Q. Gu, Z. Zhao, and D. Xiang, Phys. Rev. ST Accel. Beams 17, 090701 (2014).

[26] E. Hemsing, M. Dunning, C. Hast, T. O. Raubenheimer, S. Weathersby, and D. Xiang, Phys. Rev. ST Accel. Beams 17, 070702 (2014).

[27] E. Hemsing, M. Dunning, B. Garcia, C. Hast, T. Raubenheimer, G. Stupakov, and D. Xiang, Nat. Photonics 10, 512 (2016).

[28] F. H. Chao, C. H. Chen, Y. C. Huang, and P. J. Chou, Phys. Rev. ST Accel. Beams 18, 051301 (2015).

[29] Y. Gao, L. Feng, J. Hao, S. Huang, L. Lin, X. Luo, S. Quan, F. Wang, Z. Wang, K. Liu, and J. Chen, in Proceedings of International Particle Accelerator Conference, Richmond, USA, 2015 (JACow, Richmond, VA, 2015).

[30] A. Angelovski, M. Kuntzsch, M. K. Czwalinna, A. Penirschke, M. Hansli, C. Sydlo, V. Arsov, S. Hunziker, H. Schlarb, M. Gensch, V. Schlott, T. Weiland, and R. Jakoby, Phys. Rev. ST Accel. Beams 18, 012801 (2015).

[31] C. Gulliford, A. Bartnik, I. Bazarov, L. Cultrera, J. Dobbins, B. Dunham, F. Gonzalez, S. Karkare, H. Lee, H. Li, Y. Li, X. Liu, J. Maxson, C. Nguyen, K. Smolenski, and Z. Zhao, Phys. Rev. ST Accel. Beams 16, 073401 (2013).

[32] M. Ferrario et al., Nucl. Instrum. Methods Phys. Res., Sect. A 637, S43 (2011).

[33] D. Yu. Shchegolkov and E. I. Simakov, Phys. Rev. ST Accel. Beams 17, 041301 (2014).

[34] M. Cornacchia and P. Emma, Phys. Rev. ST Accel. Beams 5, 084001 (2002).

[35] P. Emma, Z. Huang, K.-J. Kim, and P. Piot, Phys. Rev. ST Accel. Beams 9, 100702 (2006).

[36] D. Xiang and A. Chao, Phys. Rev. ST Accel. Beams 14, 114001 (2011). 
[37] Y. E. Sun, P. Piot, A. Johnson, A. H. Lumpkin, T. J. Maxwell, J. Ruan, and R. Thurman-Keup, Phys. Rev. Lett. 105, 234801 (2010).

[38] M. J. de Loos, S. B. van der Geer, Y. M. Saveliev, V. M. Pavlov, A. J. W. Reitsma, S. M. Wiggins, J. Rodier, T. Garvey, and D. A. Jaroszynski, Phys. Rev. ST Accel. Beams 9, 084201 (2006).

[39] K. Floettmann, ASTRA, A Space Charge Tracking Algorithm DESY, Notkestrasse 85, 22603 Hamburg, Germany (Version 3.0, October 2011).

[40] K. J. Kim, Nucl. Instrum. Methods Phys. Res., Sect. A 275, 201 (1989).

[41] K. Floettmann, Phys. Rev. ST Accel. Beams 18, 064801 (2015).

[42] D. T. Palmer, X. J. Wang, R. H. Miller, M. Babzien, I. Ben-Zvi, C. Pellegrini, J. Sheehan, J. Skaritka, H. Winick, M. Woodle, and V. Yakimenko, in Proceedings of the Particle Accelerator Conference, Vancouver, BC, Canada, 1997 (IEEE, New York, 1997), p. 2687.

[43] A. P. Potylitsyn, M. I. Ryazanov, M. N. Strikhanov, and A. A. Tishchenko, Diffraction Radiation from Relativistic Particles (Springer, Berlin, 2010).

[44] J. W. Goodman, Introduction to Fourier Optics, 3rd ed. (Roberts \& Company Publishers, Englewood, 2005).

[45] A. A. Tseng, K. Chen, C. D. Chen, and K. J. Ma, IEEE Trans. 26, 141 (2003).

[46] C. Vieu, F. Carcenac, A. Pepin, Y. Chen, M. Mejias, A. Lebib, L. Manin-Ferlazzo, L. Couraud, and H. Launois,
Appl. Surf. Sci. 164, 111 (2000), https://ui.adsabs.harvard .edu/abs/2000ApSS..164..111V/abstract.

[47] Z. Zhang and C. Tang, Phys. Rev. ST Accel. Beams 18, 053401 (2015).

[48] C. X. Wang, K. J. Kim, M. Ferrario, and A. Wang, Phys. Rev. ST Accel. Beams 10, 104201 (2007).

[49] V. Kumar, Am. J. Phys. 77, 737 (2009).

[50] S. P. Weathersby et al., Rev. Sci. Instrum. 86, 073702 (2015).

[51] H. Lee, L. Cultrera, and I. Bazarov, Appl. Phys. Lett. 108, 124105 (2016).

[52] L. Cultrera, S. Karkare, H. Lee, X. Liu, I. Bazarov, and B. Dunham, Phys. Rev. ST Accel. Beams 18, 113401 (2015).

[53] C. M. Pierce, M. B. Andorf, E. Lu, C. Gulliford, I. V. Bazarov, J. M. Maxson, M. Gordon, Y.-K. Kim, N. P. Norvell, B. M. Dunham, and T. O. Raubenheimer, Phys. Rev. ST Accel. Beams 23, 070101 (2020).

[54] J. M. Maxson, I. V. Bazarov, W. Wan, H. A. Padmore, and C. E. Coleman-Smith, New J. Phys. 15, 103024 (2013).

[55] E. A. Nanni1, W. R. Huang, K.-H. Hong, K. Ravi, A. Fallahi, G. Moriena, R. J. D. Miller, and F. X. Kärtner, Nat. Commun. 6, 8486 (2015).

[56] E. A. Peralta, K. Soong, R. J. England, E. R. Colby, Z. Wu, B. Montazeri, C. McGuinness, J. McNeur, K. J. Leedle, D. Walz, E. B. Sozer, B. Cowan, B. Schwartz, G. Travish, and R. L. Byer, Nature (London) 503, 91 (2013).

[57] T. Plettner and R. L. Byer, Phys. Rev. ST Accel. Beams 11, 030704 (2008). 